\title{
Impact of Climate Change on National Instability
}

\author{
Junbo Lu \\ School of North China Electric Power University, Baoding 071000, China \\ lu_ncepu@126.com
}

Keywords: Fuzzy evaluation; climate change; extreme rainfall.

\begin{abstract}
Nowadays, the connection between a country's instability and the climate change and is getting more and more people's attention gradually. For this problem, we propose an easy model to discuss the relationship theoretically. Due to the affecting factors are uncertain, we try to take the fuzzy evaluation model into consideration to describe the phenomenon which is corresponding to the variable factors. At the beginning, we divide the country instability into three levels, respectively unstable, normal and stable. To determine the instability of a country, we select five criteria, extreme rainfall, economy, public services, human rights and refugees as the influential factors. The refined fuzzy evaluation approach based on the criteria calculation is applied. As a result, the extreme rainfall which is related to the climate change causes indirect influence to the instability of a country. As for the economy and refugee and so on, they influence the instability of a country in different levels. To analysis whether the model presented can be applied to the smaller or lager "countries", three countries of different scales of area are taken as the study targets. Supposing the indices about the climate change are basically the same, we use the model to calculate the instability of the three countries, and the results show the model may not work on well in smaller "countries" or larger "countries". It may be improved by taking some more criteria which includes more characteristic of the country into account.
\end{abstract}

\section{Introduction}

In the present moment, climate change is one of the most controversial issue in the world and may cause threats to the stability of a country. The effects of the climate change, such as the rising sea level, devastating droughts, sea level rise, global warming and resource shortage, will change people's lifestyle in daily life. Under such circumstances, people will breed conflicts and what's worse, the society will become unstable. And it is of high probability to cause the destruction of the social and governmental structures.

In most situations, climate change is interacted with the existing pressures, particularly the economic inequality, large-scale migration and the lack of resources. If it faced with these issues together, the likelihood of instability of a country will rise. Taking Syria as an example, the long-term drought problem triggers the latent social and politician crisis, aggravating the fragility of the two states. In view of those problems, more and more people are engaged in the study of state instability and try to recognize the connection between climate change and the fragility of a country.

\section{Some Assumptions}

1.The target country is not affected by any other country. It is in a relatively safe and stable environment.

2.The reference criteria is not changed in a short period. It can assure the construction of the model is right and can be tested.

3.The policies in the country is not changed in the short period.

\section{Symbol Explanation}

The symbols used in the essay is listed as follows: 
Table 1. Framework of measurable indexes

\begin{tabular}{c|c}
\hline Criteria & Explanation \\
\hline Economy: $U_{1}$ & It represents the development of a society. \\
\hline Public Services: $U_{2}$ & It refers to the medical care, education, security, etc. \\
\hline Human Rights: $U_{3}$ & It refers to the freedom of speaking, faith, etc. \\
\hline Refugees : $U_{4}$ & It represents the migrant people because of disasters. \\
\hline Extreme rainfall: $U_{5}$ & It refers to the too much or little rainfall in a unit time. \\
\hline
\end{tabular}

\section{Fuzzy Evaluation}

After the selection of the criteria, we need to identify the weight of each index. We consider that the importance of every criteria is a relative concept. So, we can only determine the relative effect of the criteria for a fragile state. According to this, we propose the fuzzy evaluation method to establish the model.

We use the five indexes Ui shown above as the element set, determining the different effect to the instability of a state. The five elements are given four judgement norms by experts, respectively $\mathrm{S} 1=10, \mathrm{~S} 2=7, \mathrm{~S} 3=5$ corresponding to "unstable, normal and stable"

$$
U=\left\{U_{1}, U_{2}, U_{3}, U_{4}, U_{5}\right\} S=\left\{S_{1}, S_{2}, S_{3}\right\}
$$

For a specific country, we have a column vector consist of five indexes. Here we introduce a conception of "Membership Degree". Since the score of each index is fuzzy, it is reasonable to use the membership degree to divide the classification threshold. The degree of membership is determined by the calculation of membership functions. As the data distribution is a matrix with a dimension $5 \times 1$. According to the 5 criteria, we have 5 membership functions according to [1].

We put the score for the 5 indexes into the previously identified membership function and can calculate the membership degree. Then we get a fuzzy evaluation matrix $\mathrm{R}$ with a dimension $5 \times 3$. By analyzing scoring data, it is found that when different experts tend to score a certain criterion for the same state, we can see that if the experts have the same rating on the criteria, the criteria are more accurate. So, the weight should be of more proportion. The criteria are vaguer in character and its weight should be smaller.

To analyses the importance of the 5 criteria, we consider giving them different weights. When an index of the score is not in an agreement, it is given a lower weight and vice versa. Based on this point, we normalize the weights. Namely, the formula stated according to [2]:

$$
w_{j}=\frac{w_{j}{ }^{\prime}}{\sum_{j=1}^{m} w_{j}{ }^{\prime}}, j=1,2, \mathrm{~L}, m
$$

After we determine the fuzzy evaluation matrix R and the weight matrix A, we can make a fuzzy comprehensive evaluation matrix $\mathrm{B}$ of the index.

$$
A=\left\{w_{1}, w_{2}\right\} \quad B=A-R
$$

In terms of the matrix $B$ and the principle of maximum membership degree, we can identify the instability for a country by the maximum value of the four-membership degree.

\section{Analysis}

To verify the reliability of our model, we choose 3 representative countries respectively Zambia, Bangladesh and Algeria. The score of the 5 indexes is found from the Fragile State Index and available information on the websites. To conclude the instability of the 3 states, the data is applied to the model. And the final result is listed in the following: 
Table 2. The membership degree of 3 countries

\begin{tabular}{c|c|c|c}
\hline Zambia & Bangladesh & Algeria & Class \\
\hline 0.879049 & 0.941351 & 0.801091 & Unstable \\
\hline 0.221567 & 0.117297 & 0.208153 & Normal \\
\hline 0.120951 & 0.0586485 & 0.104076 & Stable \\
\hline
\end{tabular}

According to the data in Table 2, we can find out the maximum data in a column for a state. The corresponding class is the final determination instability for a country. So, we conclude that the states in class fragile are Zambia, Bangladesh, Rwanda and the states in class vulnerable are Cambodia, Algeria.

\section{Directly or Indirectly}

In order to explore the climate change effects are through direct means or indirect means, we need to know the weight of the five criteria.

Table 3. The weight of the five indexes

\begin{tabular}{c|c|c|c|c|c}
\hline Index & $U_{1}$ & $U_{2}$ & $U_{3}$ & $U_{4}$ & $U_{5}$ \\
\hline Weight & 0.2246 & 0.1965 & 0.1979 & 0.2073 & 0.1737 \\
\hline
\end{tabular}

From Table 3 above we can clearly see the weight of index more than 0.2. First, in our calculation, the sum of the weights is 1 . In the ideal situation, the average weight for each index is 0.2 , and we consider the criteria more than 0.2 affects more in a state fragility. As for the climate related criteria, $U_{5}$ gets 0.1737 . In conclusion, the extreme rainfall may cause an indirect affect to a state's fragility.

\section{Application in Different Regions}

To analysis whether our model can be applied to the smaller or lager "states", we choose 3 "states" to calculate their fragility. They are respectively India, Sri Lanka, and Maldives. They are ranked the 73th, 48th, 87th in the Fragility State Index. Because the 3 states are next to each other, we consider the index about the climate change are basically the same. Similarly, we apply our model to calculate the fragility of the three states, and the results are listed in the following:

Table 4. The membership degree of the three states

\begin{tabular}{c|c|c|c}
\hline India & Sri Lanka & Maldives & Class \\
\hline 0.6780 & 0.6626 & 0.7240 & Unstable \\
\hline 0.5262 & 0.5558 & 0.4643 & Normal \\
\hline 0.3220 & 0.3374 & 0.2760 & Stable \\
\hline
\end{tabular}

We conclude that both India and Sri Lanka are in the class instability which is correspond to the actual ranking. Unluckily, Maldives is in the class instability which is against the reality. It reveals that our model may not work on well for smaller "states" or larger "states".

\section{References}

[1]. Zhu Xiao-Fei, Wang Yong-Jun, LI Da-Jun. Analysis of the Validity of the Principle of Maximum Membership in Fuzzy Evaluation [J]. Metrics \& Mapping, 2016,5 (5): 135-137,143. DOI: 10.3969 / j. issn.1672-5867.2016.05.039.

[2]. Lan Jibin, Xu Yang, Huo Liangan, et al. Research on the priorities of fuzzy analytical hierarchy process[J]. Systems Engineering Theory \& Practice,2006,26(9) :107-112.

[3]. Liu Tianxu, Wu Tao. Evaluation Criteria for Fragile Countries [J]. Leading Science Forum, 2016 (13): 17-26. 
[4]. Reservoir Operation Comprehensive Evaluation Model Based on Improved Set Pair Analysis [J]. Ren Bingjun, Sun Yichao, Zhou Zhengyin, Cheng Zhengfei, Hu Xingfu. Journal of Tianjin University 2013(01).

[5]. Study on Low-carbon Policy Multi-criteria Evaluation in Liaoning Province Based on Fuzzy Analytic Hierarchy Process[D]. Tian Lu. Dongbei University of Finance and Economics 2011. 